\title{
O CONCEITO DE INCERTEZA APLICADO AOS PROCESSOS DE MEDIÇÃO ASSOCIADOS À PREPARAÇÃO DE UMA SOLUÇÃO DE REFERÊNCIA PARA CALIBRAÇÃO
}

\author{
José Henrique Buchmann e Jorge Eduardo de Souza Sarkis
}

Instituto de Pesquisas Energéticas e Nucleares, Travessa R, 400, 05508-900 São Paulo - SP

Recebido em 6/11/00; aceito em 25/7/01

\begin{abstract}
THE UNCERTAINTY CONCEPT AS APPLIED TO THE MEASUREMENT PROCESS ASSOCIATED WITH THE PREPARATION OF A CALIBRATION REFERENCE SOLUTION. The implementation of a quality assurance program in chemical analytical laboratories, that can aid in demonstrate the quality of their results, is an issue of great concern. As a consequence, it is mandatory to give an estimate of the confidence that can be placed on the obtained results. An useful measure of this confidence is the measurement uncertainty and, nowadays, a result without the corresponding uncertainty statement cannot be considered reliable. This paper presents a summary of the most important mechanisms for the evaluation and reporting of the measurement uncertainty. In implementing these principles, it is described the measurement uncertainty estimation associated with the preparation of a uranium elemental reference solution at $2.4 \mathrm{mg} \cdot \mathrm{kg}^{-1}$ from the corresponding certified reference material (in this example at $\left.1003 \mathrm{mg} \cdot \mathrm{kg}^{-1}\right)$.
\end{abstract}

Keywords: uncertainty; quality assurance; chemical metrology.

\section{INTRODUÇÃO}

Paulatinamente, a implementação de um programa de garantia da qualidade em um laboratório de química analítica torna-se mais um requisito fundamental para a conquista de novos mercados e para a oferta de serviços especializados com qualidade comprovada.

A Figura 1 apresenta, esquematicamente, um enfoque típico da estrutura de um programa de garantia da qualidade que pode ser implantado em uma instituição ${ }^{1}$.

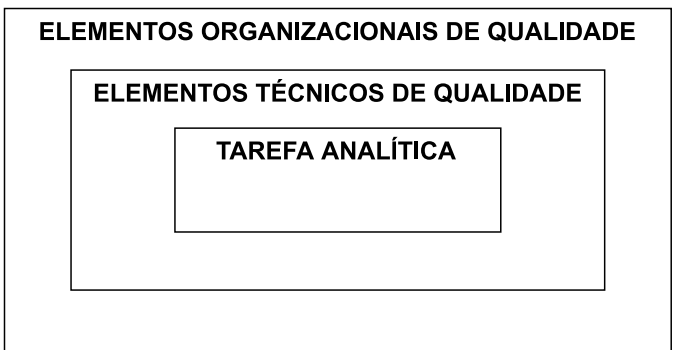

Figura 1. Estrutura das atividades de um programa de garantia da qualidade.

A Tabela 1 apresenta as várias aplicações dos materiais de referência nos laboratórios de química analítica ${ }^{2}$. É através dos materiais de referência $\left[\mathrm{VIM}^{3} 6.13\right]^{\mathrm{i}}$ que são estabelecidas a rastreabilidade e a calibração [VIM 6.10, 611].

A calibração estabelece a forma com que o sistema responde a alterações no parâmetro medido, enquanto que a rastreabilidade estabelece a exatidão do resultado da medição [VIM 3.5] através de materiais de referência.
Tabela 1. Aplicações dos materiais de referência nos sistemas de medição.

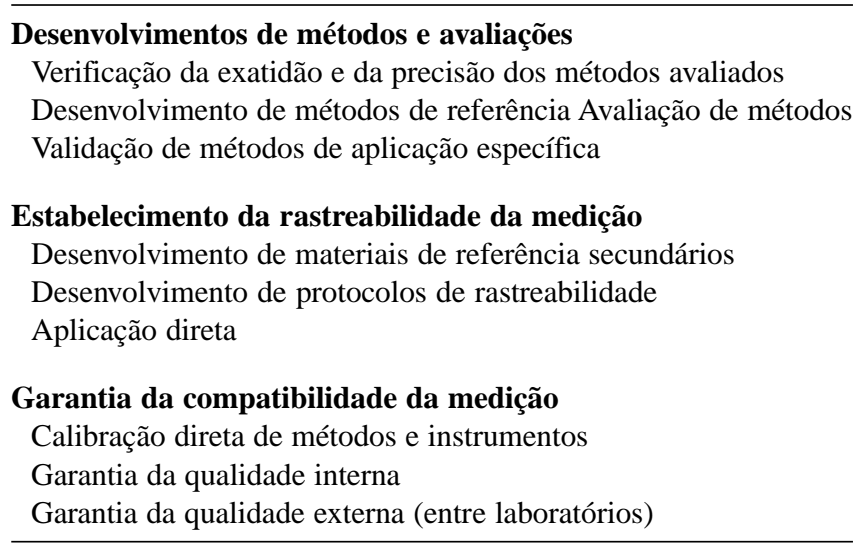

Essencialmente, a rastreabilidade é uma propriedade do valor do resultado de uma medição ou do valor transportado por um material de referência, nunca podendo ser atribuída a um método, a um instrumento, a um material ou a um Instituto (Nacional) de Metrologia, mas sempre a outro valor ${ }^{4}$.

As técnicas estatísticas, por sua vez, são ferramentas indispensáveis no desenvolvimento e na utilização dos procedimentos de medição [VIM 2.5]. A caracterização do desempenho de um determinado procedimento de medição (validação) e a estimativa [ISO $\left.3534-1^{5}, 2.51\right]^{\mathrm{ii}}$ da incerteza a ele associada utilizam as mesmas ferramentas 6 .

O "Guia", publicado de forma conjunta pela ISO e outras entidades $^{7}$, apresenta as regras gerais para a expressão da incerteza. Es-

\footnotetext{
${ }^{i}$ As definições dos termos metrológicos relevantes utilizadas neste trabalho foram extraídas do "International vocabulary of basic and general terms in metrology" (VIM), sendo a cláusula na qual o termo está definido indicada entre colchetes.

ii As definições dos termos estatísticos básicos relevantes utilizados neste trabalho foram extraídas do "International Organization for Standardization 3534l" (ISO 3534-1), sendo a cláusula na qual o termo está definido indicada entre colchetes.
} 
ses princípios são adaptados para a aplicação em metrologia química num documento distribuído pela EURACHEM ${ }^{8}$. Nesse documento, são apresentados os passos necessários para a avaliação das incertezas associadas aos processos de medição química. Na implementação desses princípios, é de fundamental importância a existência de práticas analíticas capazes de subsidiar as informações necessárias à avaliação dessas incertezas.

Apesar de alguns trabalhos relativos a esse tipo de enfoque em química analítica já terem sido publicados ${ }^{9-12}$, poucos apresentam aplicações práticas dessa metodologia que contemplem o detalhamento da identificação, avaliação e combinação das incertezas provenientes dos diversos fatores que compõem a incerteza global da medição ${ }^{13,14}$.

\section{Fontes de incerteza em metrologia química}

As metas de um laboratório de química analítica são estabelecidas na sua Política de Qualidade. A rastreabilidade e a incerteza são, atualmente, os mais importantes elementos do sistema de garantia da qualidade implementado nos laboratórios de química analítica.

A avaliação da incerteza, realizada de acordo com os princípios estabelecidos pela $\mathrm{ISO}^{7}$, é baseada na identificação e quantificação dos efeitos dos parâmetros de influência sobre a incerteza global. Além disso requer a compreensão do procedimento de medição e das incerteza associadas a cada um dos fatores que influenciam o resultado.

O termo "incerteza" está intimamente relacionado a "dúvida". A incerteza de medição nada mais é do que uma dúvida com relação ao valor obtido durante o procedimento de medição. Basicamente, as fontes de incerteza em metrologia química podem ser provenientes de definições incompletas (do mensurando [VIM 2.6]), da amostragem, de efeitos de matriz e interferências, de condições ambientais, das pesagens e volumetria e de aproximações incorporadas aos métodos [VIM 2.4] e procedimentos de medição (por exemplo, a utilização de curvas de calibração).

Normalmente, o resultado de uma medição [VIM 3.1] ou do cálculo de uma grandeza representa somente uma aproximação, ou uma estimativa do valor do mensurando e, portanto, somente é completo quando acompanhado de uma declaração da incerteza associada.

A medição de uma grandeza é sempre iniciada através da identificação do mensurando, associado aos respectivos método de medição e procedimento de medição.

Com o objetivo de identificar e analisar as possíveis fontes de incerteza na medição [VIM 2.1] das grandezas avaliadas, os procedimentos adotados em cada etapa devem ser detalhados e estruturados de forma a garantir a máxima abrangência, evitando a duplicidade de fontes de incerteza. Normalmente, este objetivo pode ser alcançado e melhor visualizado através da utilização de diagramas de causa e efeito, também conhecidos como diagramas de Ishikawa ou de espinha de peixe ${ }^{2,8}$.

Antes de combinar todas as contribuições de incerteza para a obtenção da incerteza global do mensurando, essas devem ser expressas na forma de incertezas padrão, ou seja, como desvio padrão [ISO 3534-1, 1.23, 2.34]. A incerteza padrão proveniente de efeitos aleatórios é, normalmente, estimada a partir de uma série de experimentos em replicata, sendo sua estimativa quantificada em termos do desvio padrão dos valores medidos. Segundo recomendações do "Working Group on the Statement of Uncertainties" ${ }^{15}$, o método de avaliação de incerteza utilizado nesse caso é denominado como sendo do Tipo A. O método de avaliação é considerado Tipo B quando essa avaliação é realizada através de um julgamento científico, baseado em todas as informações possíveis sobre a variabilidade da grandeza medida. Torna-se, portanto, necessário quantificar cada fonte potencial de incerteza através de sua medição direta, de sua avalia- ção a partir de dados experimentais obtidos previamente ou, então, através de sua dedução a partir de análises teóricas.

Define-se mensurando, genericamente denominado $Y$, como sendo uma grandeza específica [VIM 1.1, Nota 1] submetida a uma medição. Na maioria dos casos, o valor do mensurando não é medido diretamente, mas é determinado a partir de $N$ outras grandezas $X_{i}$, através da função $Y=f\left(X_{1}, X_{2}, \ldots, X_{N}\right)$.

Para uma determinada variável aleatória [ISO 3534-1, 1.2] $X_{i}$, avaliada por $n$ observações independentes $X_{i, k}$, a média aritmética [ISO 3534-1, 2.26] $\bar{X}_{i}$, obtida através da equação,

$$
\bar{X}_{i}=\frac{1}{n} \sum_{k=1}^{n} X_{i, k}
$$

é usada como a melhor estimativa $x_{i}$ de sua esperança [ISO 35341, 1.18] ou valor esperado. A melhor estimativa $y$ do mensurando $Y$ será obtida, portanto, através da relação funcional $y=f\left(x_{1}, x_{2}, \ldots, x_{N}\right)$.

A variância [ISO 3534-1, 1.22, 2.33] experimental das observações $X_{i, k}$ representa uma estimativa da variância $\sigma^{2}$ da função de distribuiçã̃o de probabilidade [ISO 3534-1, 1.3] de $X_{i, k}$ e é obtida através da relação:

$$
s^{2}\left(X_{i, k}\right)=\frac{1}{n-1} \sum_{k=1}^{n}\left(X_{i, k}-\bar{X}_{i}\right)^{2} .
$$

A raiz positiva de $s^{2}\left(X_{i, k}\right)$, denominada desvio padrão experimental $s\left(X_{i, k}\right)$ [VIM 3.8], caracteriza a variabilidade dos valores observa$\operatorname{dos} X_{i, k}$ em torno de sua média $\bar{X}_{i}$.

A melhor estimativa de $\sigma^{2}\left(\bar{X}_{i}\right)=\sigma^{2} / n$, a variância experimental da média, é dada por:

$$
s^{2}\left(\bar{X}_{i}\right)=\frac{s^{2}\left(X_{i, k}\right)}{n} .
$$

A variância experimental da média e a sua raiz quadrada positiva, o desvio padrão experimental da média [VIM 3.8, nota 2], quantificam o afastamento entre $\bar{X}_{i}$ e seu valor esperado $\mu_{X i}$ e, conseqüentemente, podem ser usados como uma estimativa da incerteza de $\bar{X}_{i}$. Nos casos em que a estimativa $x_{i}$ de uma grandeza $X_{i}$ não foi obtida a partir de experimentos em replicata, a variância $u^{2}\left(x_{i}\right)$ ou a incerteza padrão $u\left(x_{i}\right)$ são avaliadas através de julgamento científico com base nas informações disponíveis a cerca da variabilidade de $X_{i}$. Trata-se de uma avaliação do Tipo B da incerteza padrão.

No caso particular em que o mensurando envolve apenas a soma ou a diferença de grandezas, isto é, $Y=q\left(X_{1}+X_{2}+\ldots X_{N}\right)$, onde $q$ é uma constante, a variância combinada é expressa por ${ }^{8}$ :

$$
u_{c}^{2}(y)=q^{2} \times \sum_{i=1}^{N} u^{2}\left(x_{i}\right) .
$$

No caso particular em que a função representada pelo mensurando assume a forma dada pela relação $Y=q X_{1}^{p_{1}} X_{2}^{p_{2}} \ldots X_{N}^{p_{N}}$, onde os expoentes $p_{i}$ são números conhecidos, positivos ou negativos e possui incertezas desprezíveis, a variância combinada é expressa por?

$$
\left[\frac{u_{c}(y)}{y}\right]^{2}=q^{2} \times \sum_{i=1}^{N}\left[\frac{p_{i} \times u_{i}\left(x_{i}\right)}{x_{i}}\right]^{2} .
$$

A incerteza expandida $U$ é obtida através da multiplicação da incerteza combinada $u_{c}(y)$ por um fator de abrangência $\mathrm{k}$.

$$
U=k u_{c}(y)
$$

O valor do fator de abrangência $k$ é escolhido com base no nível da confiança requerida para o intervalo $y-U$ até $y+U$. Em geral, é recomendado que o valor de esteja situado entre 2 e 3 . Portanto, o resultado de uma medição é convenientemente expresso por $Y=y \pm U$. 
A seguir, é descrita uma aplicação prática da metodologia proposta por essas organizações à avaliação das incertezas em metrologia [VIM 2.2] química.

\section{Avaliação das incertezas associadas ao procedimento de preparação de uma solução de referência para calibração}

Com a finalidade de ilustrar a aplicação dos conceitos anteriormente descritos, será apresentada a sistemática adotada na preparação de uma solução de referência elementar, utilizada em métodos de medição para a determinação do conteúdo elementar em amostras através de análise quantitativa.

O método de calibração externa, utilizado nas análises de concentrações do tipo elementar, requer a medição de um conjunto de soluções de referência de concentrações conhecidas dos analitos de interesse. Essas soluções são preparadas a partir de soluções estoque elementares com concentração certificada, adquiridas de um fornecedor comercial (Spex ${ }^{\circledR}$ Chemical, Metuchen, NJ, EUA). A concentração elementar de cada uma delas situa-se em torno de $1000 \mathrm{mg} \cdot \mathrm{kg}^{-1}$, com uma incerteza de $\pm 0,5 \%$ do valor declarado no certificado de análise. Para a avaliação da incerteza, é necessário um exame detalhado do procedimento de medição. A Figura 2 apresenta os passos utilizados durante o desenvolvimento deste trabalho envolvidos nessa atividade ${ }^{6,8}$.

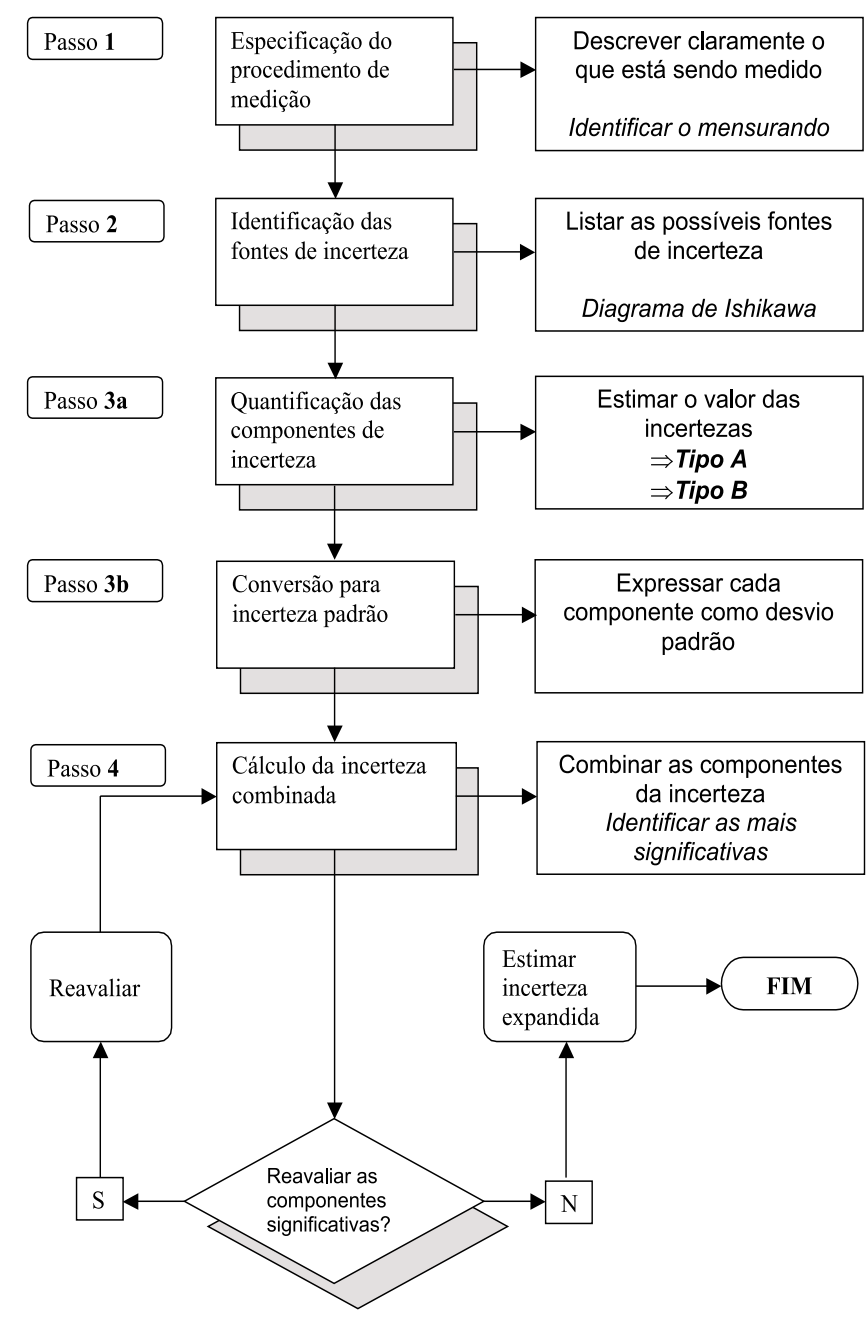

Figura 2. Sistemática adotada para a avaliação da incerteza da medição.

\section{Especificação do procedimento de medição}

A Figura 3 apresenta as etapas constituintes do procedimento adotado para a preparação de $100 \mathrm{~g}$ de solução de referência elementar de urânio na concentração de $2,4 \mathrm{mg} \cdot \mathrm{kg}^{-1}$.

No caso da concentração dessa solução, o mensurando é determinado através da relação:

$$
C_{2,4 m g \cdot k g^{-1}}=\frac{C_{\text {Spex }} \times m_{\text {Spex }}}{m_{2,4 m g \cdot k g^{-1}}},
$$

onde $C_{2,4 m g, k g^{-1}}$ é a concentração final desejada da solução de referência elementar, $C_{S p e x}$ e $m_{S p e x}$ são, respectivamente, a concentração e a massa da solução estoque elementar de urânio Spex ${ }^{\circledR}$ adicionada a um frasco de polipropileno e $m_{2,4 \mathrm{mg}_{\mathrm{kg}} \mathrm{g}^{-1}}$ é a massa final de solução obtida. O valor de $m_{2,4 \mathrm{mg} \cdot \mathrm{kg}^{-1}}$ é obtido por diferença entre a massa total, $m_{\text {total }}$, do frasco de polipropileno cheio de solução e a sua respectiva tara, $m_{\text {tara }}$.

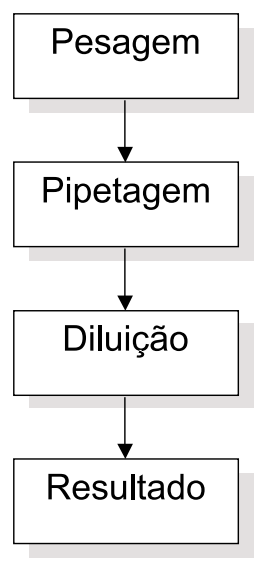

Figura 3. Preparação da solução de referência elementar de 2,4 mg. $\mathrm{kg}^{-1}$.

\section{Identificação das fontes de incerteza}

Nesta etapa, deve-se identificar as maiores fontes de incerteza associadas aos procedimentos de medição utilizados e compreender seus efeitos sobre o mensurando. A forma mais adequada de visualizar esses efeitos é a elaboração de um diagrama de causa e efeito procurando, dessa forma, evitar o uso duplicado ou a desconsideração das diversas fontes de incerteza. A Figura 4 apresenta o diagrama relativo à preparação da solução de referência elementar. Nos três ramos principais, estão colocadas as três grandezas de entrada da equação que representa o mensurando. Nos ramos secundários, estão

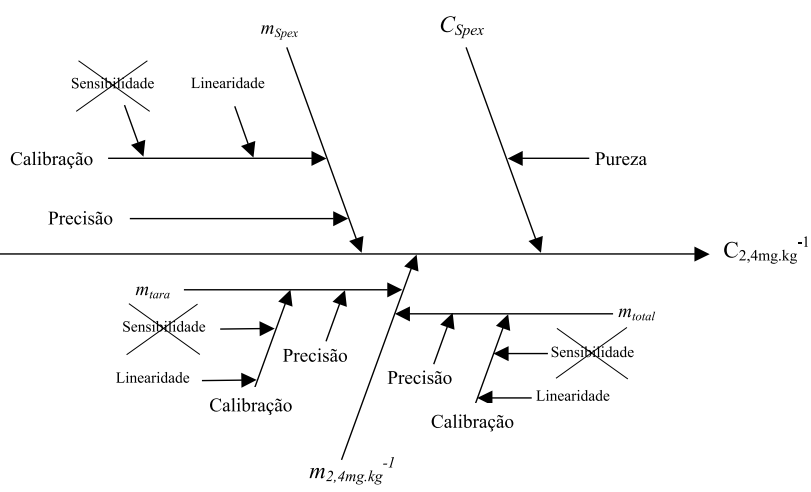

Figura 4. Diagrama de causa e efeito: solução de referência elementar de $2,4 \mathrm{mg} \cdot \mathrm{kg}^{-1}$. 
especificadas as fontes de incerteza associadas direta ou indiretamente a essas grandezas. Uma vez identificadas e analisadas as principais fontes de incerteza associadas às etapas do procedimento de preparação da solução de referência elementar, torna-se necessária a quantificação dessas incertezas. Essa quantificação é feita através da avaliação da incerteza padrão associada a cada grandeza considerada e, posteriormente, da determinação das incertezas padrão combinada e expandida.

\section{Avaliação da incerteza associada à massa da solução de referência elementar $m_{2,4 m g . k g}{ }^{-1}$}

O procedimento utilizado para a determinação da massa de solução envolveu duas etapas: a pesagem do frasco de polipropileno vazio $\left(m_{\text {tara }}\right)$ e após ter sido completado com ácido nítrico a $0,98 \mathrm{~mol} . \mathrm{kg}$ ${ }^{1}$ até a massa total equivalente a $100 \mathrm{~g}\left(m_{\text {total }}\right)$. Cada uma dessas medições está sujeita à variabilidade decorrente de pesagens sucessivas e à incerteza de calibração da balança. A calibração propriamente dita possui duas fontes de incerteza: a sensibilidade e a linearidade da balança.

A massa da solução de referência elementar foi obtida através da diferença de pesagens, ou seja, através de duas medições independentes, realizadas na mesma balança em um período curto de tempo. Portanto, a contribuição da sensibilidade à incerteza foi desprezada. A Tabela 2 apresenta o resultado das medições.

Tabela 2. Medição da massa de solução de referência elementar de urânio.

\begin{tabular}{llr}
\hline Descrição & & Valor $x_{i}(\mathrm{mg})$ \\
\hline Massa do frasco com a solução (observada) & $m_{\text {total }}$ & $146.311,52$ \\
Massa do frasco de polipropileno (observada) & $m_{\text {tara }}$ & $46.086,64$ \\
Massa de solução (calculada) & $m_{2,4 m g . k g^{-1}}$ & $100.224,88$ \\
\hline
\end{tabular}

\section{Repetibilidade}

A variabilidade do resultado da medição, obtida através de pesagens sucessivas, normalmente é fornecida no catálogo do fabricante do instrumento. Neste trabalho, a contribuição da repetibilidade na incerteza dessa medição foi sempre determinada através de um conjunto de dez pesagens sucessivas de dois pesos padrão de verificação, sendo um de massa próxima ao valor da tara (50 g) e o outro de massa próxima ao peso bruto $(100 \mathrm{~g})$. A seguir, a diferença $D$ foi calculada para cada um $\operatorname{dos} n=10$ conjuntos de 10 medidas sucessivas.

Para a grandeza $X_{1}=D$, a diferença obtida durante o processo de $n=10$ pesagens independentes $X_{1, k}=D_{k}$, a incerteza padrão $u\left(x_{i}\right)$ de sua estimativa $x_{1}=\bar{X}_{1}=\bar{D}$ é $u\left(x_{1}\right)=s\left(\bar{X}_{1}\right)=s(\bar{D})$, com $s^{2}(\bar{D})$ dado pela Equação 3. Para os valores experimentais obtidos, resultou $u\left(x_{1}\right)=0,008 \mathrm{mg}$.

\section{Calibração/linearidade}

O certificado de calibração da balança fornece um erro [VIM 3.10] de indicação equivalente a $\pm 0,42 \mathrm{mg}$ para a linearidade. Esse valor é a diferença máxima obtida entre a carga aplicada e a indicação da balança. Trata-se de uma declaração de incerteza sem informações relativas ao comportamento da variável dentro do intervalo especificado.

Quando não existem informações específicas sobre os possíveis valores da incerteza de medição dentro de um intervalo fornecido de amplitude $\pm a$, pode-se apenas considerar que é igualmente provável que o valor real de $X_{i}$ esteja em qualquer ponto situado entre - $a$ e $+a$, e que a probabilidade de que o valor real de $X_{i}$ seja encontrado fora desse intervalo é nula. Trata-se de uma distribuição de probabilidade do tipo retangular ou uniforme, para a qual a melhor estimativa do mensurando encontra-se no centro do intervalo, ou seja, $=[(-a)+(+a)] / 2$. Considerando $2 a=(+a)-(-a)$, a variância estimada associada a essa grandeza é e $^{7}$

$$
u^{2}\left(x_{i}\right)=\frac{a^{2}}{3} .
$$

Portanto, a sua estimativa deverá ser obtida através de uma avaliação do Tipo B, utilizando-se a Equação 8, logo,

$$
u\left(x_{2}\right)=\frac{0,42}{\sqrt{3}}=0,24 \mathrm{mg} .
$$

Finalmente, as duas componentes de incerteza da medição de massa, a repetibilidade e a linearidade, necessitam ser combinadas para fornecer a incerteza padrão combinada $u_{c}\left(m_{2,4 \mathrm{mg}_{\mathrm{g}} \mathrm{g}^{-1}}\right)$ da massa de solução de referência para calibração contida dentro dos frascos de polipropileno. Essa combinação foi feita através da Equação 4, onde a contribuição da repetibilidade foi considerada apenas uma vez já que o desvio padrão das diferenças entre a tara e o peso bruto foi determinado diretamente. A contribuição da linearidade foi considerada duas vezes, uma para a tara e outra para o peso bruto, logo,

$$
u_{c}\left(m_{2,4 m g \cdot k g^{-1}}\right)=\sqrt{(0,008)^{2}+2 \times(0,24)^{2}}=0,3 \mathrm{mg} .
$$

\section{Avaliação da incerteza associada à concentração da solução estoque $C_{\text {Spex }}$}

A concentração da solução estoque elementar Spex ${ }^{\circledR}$ de urânio certificada foi de $1003 \mathrm{mg} \cdot \mathrm{kg}^{-1}$. O certificado de análise declara uma incerteza igual a $\pm 0,5 \%$. Essa incerteza declarada foi avaliada através de um método do Tipo B. Como não existem informações específicas sobre a distribuição dos valores de concentração medidos dentro do intervalo especificado, considerou-se uma distribuição retangular. $\mathrm{O}$ valor da incerteza padrão foi calculado com o auxílio da Equação 8,

$$
u\left(C_{\text {Spex }}\right)=\frac{1003 \times 0,005}{\sqrt{3}}=3 \mathrm{mg} \cdot \mathrm{kg}^{-1} .
$$

\section{Avaliação da incerteza associada à massa da solução estoque}

A adição de solução estoque elementar $\operatorname{Spex}{ }^{\circledR}$ ao frasco de polipropileno, para diluição com ácido nítrico a $0,98 \mathrm{~mol} \cdot \mathrm{kg}^{-1}$ até a concentração de $2,4 \mathrm{mg} \cdot \mathrm{kg}^{-1}\left(C_{2,4 \mathrm{mg} \cdot \mathrm{kg}^{-1}}\right)$, foi feita com o auxílio de uma micropipeta modelo Finnpipette (Labsystems, Helsinki, Finlândia) de volume máximo equivalente a $1000 \mu \mathrm{l}$. As características dessa micropipeta, fornecidas pelo fabricante, encontram-se na Tabela 3. A massa de solução fornecida pela micropipeta está sujeita a duas fontes principais de incerteza: a repetibilidade da massa fornecida e a incerteza de calibração da micropipeta.

Tabela 3. Característica da micropipeta utilizada.

\begin{tabular}{lccccc}
\hline Identificação & & \multicolumn{2}{c}{ Exatidão $( \pm \%)$} & \multicolumn{2}{c}{ Precisão $( \pm \%)$} \\
\hline Série & Faixa & $\begin{array}{c}\text { Vol. } \\
\text { mínimo }\end{array}$ & $\begin{array}{c}\text { Vol. } \\
\text { máximo }\end{array}$ & $\begin{array}{c}\text { Vol. } \\
\text { mínimo }\end{array}$ & $\begin{array}{c}\text { Vol. } \\
\text { máximo }\end{array}$ \\
\hline B59434 & $200-1000 \mu 1$ & 0,60 & 0,30 & 0,90 & 0,30 \\
\hline
\end{tabular}

\section{Repetibilidade da massa fornecida}

O uso de uma micropipeta para a manipulação de pequenas quantidades de solução, em geral, não garante o completo fornecimento/ 
Tabela 4. Valores e incertezas da solução de referência elementar de urânio.

\begin{tabular}{clccc}
\hline \multicolumn{1}{c}{ Descrição } & & Valor & Incerteza padrão & Incerteza padrão relativa \\
& & $x_{i}$ & $u\left(x_{i}\right)$ & $u\left(x_{i}\right) / x_{i}$ \\
\hline Massa a 2,4 mg.kg-1 & $m_{2,4 m g \cdot k^{-1}}$ & $100.224,88 \mathrm{mg}$ & $0,3 \mathrm{mg}$ & 0,000003 \\
Concentração Spex & $C_{\text {Spex }}$ & $1003 \mathrm{mg} \cdot \mathrm{kg}^{-1}$ & $3 \mathrm{mg} \cdot \mathrm{kg}^{-1}$ & 0,003 \\
Massa padrão Spex & $m_{\text {Spex }}$ & $241,73 \mathrm{mg}$ & $0,34 \mathrm{mg}$ & 0,0014 \\
\hline
\end{tabular}

esgotamento de seu conteúdo. Para avaliar a variabilidade de uma determinada quantidade fornecida pela micropipeta é necessário considerar as diversas faixas nas quais essa quantidade possa estar situada. $\mathrm{O}$ certificado de calibração da micropipeta (Tabela 3) fornece um valor para a precisão da medição [ISO 3534-1, 3.14], [VIM 3.5, Nota 2] e, conseqüentemente, permite avaliar a incerteza associada à repetibilidade através de um método do Tipo B. Neste trabalho, a repetibilidade foi verificada antecipadamente através de uma operação repetitiva de dez enchimentos, fornecimentos e pesagens sucessivas. A estimativa de uma quantidade equivalente a $240 \mathrm{mg}$, calculada de acordo com a Equação 1, resultou $\bar{m}_{\text {Spex }}=241,73 \mathrm{mg}$. Dessa forma, a incerteza padrão $u\left(x_{l}\right)$ da estimativa $x_{1}=\bar{X}_{1}=\bar{m}_{\text {Spex }}$ da grandeza $\quad X_{1}=m_{\text {Spex }}$, avaliada por um processo de $n=10$ pesagens independentes, é $u\left(x_{l}\right)=s\left(\bar{X}_{1}\right), \operatorname{com} s^{2}\left(\bar{X}_{l}\right)$ dado pela Equação 3. Para os valores experimentais obtidos, resultou $u\left(x_{1}\right)=$ $0,333 \mathrm{mg}$.

\section{Calibração/Linearidade}

Os limites de exatidão da medição [VIM 3.5] da massa fornecida pela micropipeta utilizada também estão declarados no seu certificado de calibração (Tabela 3). Contudo, procurou-se determinar esse valor de uma forma independente, através das operações utilizadas para a avaliação da repetibilidade. Para cargas aplicadas na balança utilizada até um limite máximo igual a $1 \mathrm{~g}$, o certificado de calibração fornece um erro máximo de indicação igual a $\pm 0,12 \mathrm{mg}$. Procedendo-se à avaliação da estimativa dessa incerteza por um método do Tipo B e, considerando-se uma distribuição retangular, a incerteza padrão obtida através da Equação 8, resultou $u\left(x_{2}\right)=0,12 / \sqrt{3}=0,069 \mathrm{mg}$.

$\mathrm{O}$ valor encontrado para $m_{\text {spex }}$ foi $241,73 \mathrm{mg}$. Combinando as duas contribuições à incerteza $u\left(m_{\text {Spex }}\right)$ da massa $m_{\text {Spex }}$ através da Equação 4 , resultou $u_{c}\left(m_{\text {Spex }}\right)=\sqrt{0,333^{2}+0,069^{2}}=0,34 \mathrm{mg}$. Se, ao invés de determinar de uma forma independente as incertezas decorrentes da repetibilidade e da linearidade, tivéssemos utilizado uma distribuição retangular com os valores declarados pelo fabricante, conforme Tabela 3, na melhor das hipóteses, teríamos:

$$
u_{c}\left(m_{\text {Spex }}\right)=\sqrt{\left(\frac{0,73}{\sqrt{3}}\right)^{2}+\left(\frac{0,73}{\sqrt{3}}\right)^{2}}=0,59 \mathrm{mg},
$$

que é um valor $74 \%$ superior ao obtido experimentalmente.

Para exemplificar a forma de obtenção dos resultados para a solução de referência elementar de urânio, apresentamos na Tabela 4 os valores de todas as variáveis utilizadas na Equação 7, bem como suas incertezas padrão e suas incertezas padrão relativas.

Utilizando-se esses valores, a concentração da solução de referência elementar de urânio calculada foi $C_{u}=2,42 \mathrm{mg} \cdot \mathrm{kg}^{-1}$.

Com o objetivo de combinar as incertezas associadas a cada um dos componentes da expressão multiplicativa da Equação 7, tornase necessária a utilização da Equação 5.

Utilizando-se, novamente, os valores apresentados na Tabela 4, a incerteza combinada da concentração da solução padrão intermediária de urânio é dada por:

$$
u_{c}\left(C_{u}\right)=2,42 \times \sqrt{0,000003^{2}+0,003^{2}+0,0014^{2}},
$$

resultando $u_{c}\left(C_{u}\right)=0,01 \mathrm{mg} \cdot \mathrm{kg}^{-1}$. Portanto, a concentração da solução de referência de urânio para calibração $C_{u}=2,42 \mathrm{mg} \cdot \mathrm{kg}^{-1}$, preparada a partir da solução estoque elementar Spex ${ }^{\circledR}$, tem uma incerteza padrão combinada de $u_{c}\left(C_{u}\right)=0,01 \mathrm{mg} \cdot \mathrm{kg}^{-1}$.

$\mathrm{O}$ resultado final do valor da concentração da solução de referência elementar obtido e das correspondentes incertezas globais estimadas encontram-se apresentados na Tabela 5.

Tabela 5. Concentração da solução de referência elementar de urânio para calibração.

\begin{tabular}{ccc}
\hline $\begin{array}{c}\text { Concentração } \\
C\end{array}$ & $\begin{array}{c}\text { Incerteza combinada } \\
u_{c}(C) \\
\left(\mathrm{mg} \cdot \mathrm{kg}^{-1}\right)\end{array}$ & $\begin{array}{c}\text { Incerteza expandida } \\
\left(\mathrm{mg} \cdot \mathrm{kg}^{-1}\right)\end{array}$ \\
\hline 2,42 & 0,01 & $\begin{array}{c}U(C) \\
\left(\mathrm{mg} \cdot \mathrm{kg}^{-1}\right)\end{array}$ \\
\hline
\end{tabular}

${ }^{a}$ Resultante do produto da incerteza combinada por um fator de abrangência k=2, para um nível da confiança de aproximadamente $95 \%$.

Finalmente, foi possível examinar as contribuições relativas das diferentes grandezas de entrada à incerteza padrão combinada. A Figura 5 apresenta, através de um histograma das incertezas padrão relativas, a influência da incerteza de medição de cada grandeza de entrada na incerteza global. $C_{2,4 \mathrm{mg} \cdot \mathrm{kg}^{-1}}$ representa a incerteza padrão combinada relativa $(0,0032)$ da concentração final, obtida para a solução de referência elementar.

A contribuição da incerteza associada à concentração da solução estoque elementar $\operatorname{Spex}^{\circledR}$ foi a maior de todas, seguida pela associada à quantidade de padrão pipetado durante a diluição. A incerteza envolvida no processo de pesagem da solução final, praticamente não teve influência sobre a incerteza global obtida.

A análise da Figura 5 revela que qualquer melhoria que se pretenda inserir no procedimento de preparação da solução de referên-

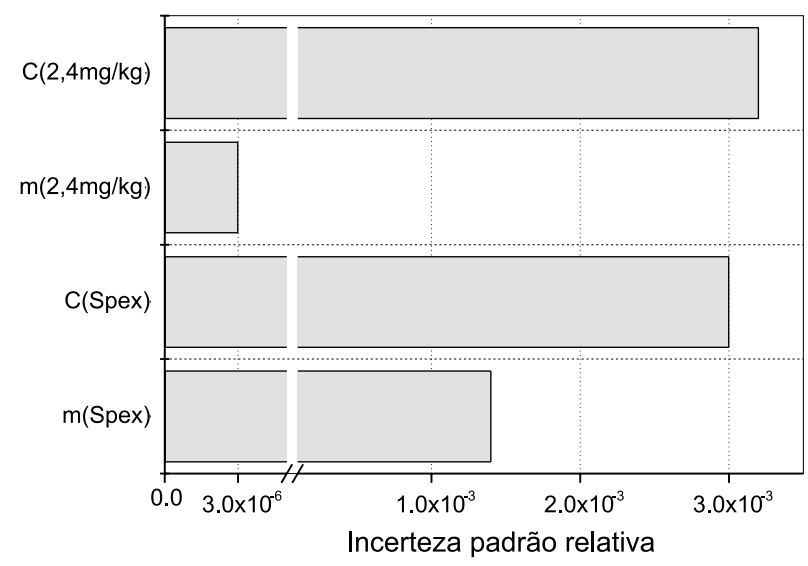

Figura 5. Contribuições das incertezas parciais na incerteza global da concentração da solução de referência elementar de urânio. 
cia elementar, com o objetivo de diminuir a incerteza global obtida, deve estar relacionada à transferência de pequenas quantidades de solução através do uso de micropipetas.

\section{CONCLUSÃO}

Este trabalho ilustra a aplicação das ferramentas estatísticas para a avaliação das incertezas em uma atividade comum nos laboratórios de química analítica, qual seja a preparação de soluções de referência elementares, utilizadas nos métodos de calibração externa.

A sistemática aplicada para a avaliação das incertezas associadas aos procedimentos de medição não requer muita mão de obra, mas uma racionalização das etapas que constituem os procedimentos analíticos. Ao longo do trabalho foram apresentadas as definições, terminologias e procedimentos de cálculo, conforme a literatu$\mathrm{ra}^{7}$, utilizados para a avaliação da incerteza global expandida, bem como para inferir qual a maior fonte de incerteza.

Neste trabalho, os resultados indicaram que a incerteza declarada para a concentração do material de referência predomina. Uma vez que não é possível alterar a declaração da incerteza do material de referência utilizado, é na segunda maior contribuição, a pipetagem, que devem ser concentrados os esforços. A redução da incerteza global estimada da solução de referência elementar obtida é possível através de um cuidado maior na execução dessa atividade.

A comparação entre o valor (1003 $\pm 5 \%) \mathrm{mg} \cdot \mathrm{kg}^{-1}$, declarado pelo fornecedor comercial (Spex ${ }^{\circledR}$ Chemical, Metuchen, NJ, EUA) e o valor $(2,42 \pm 0,02) \mathrm{mg} \cdot \mathrm{kg}^{-1}$, encontrado pelos autores, deveria permitir realizar a rastreabilidade. No entanto, a incerteza declarada pelo fornecedor comercial não foi especificada com um certo grau de confiança, razão pela qual não será possível realizar a rastreabilidade. Por consequiência, todo o usuário desta última solução não poderá rastrear até o Sistema Internacional, ficando prejudicado um dos requisitos importantes da Metrologia, constituindo-se em uma não conformidade.

As ferramentas estatísticas utilizadas partiram do pressuposto que as incertezas obedecem à Lei Normal de Distribuição. Para verificar-se a veracidade do pressuposto, é válido realizar os procedimentos recomendados pela literatura ${ }^{16}$.

\section{REFERÊNCIAS}

1. EURACHEM. Quality assurance for research and development and nonroutine analysis. Teddington, UK, 1998.

2. Taylor, J. K.; Quality assurance of chemical measurements; Lewis Publishers; Florida, 1987.

3. INTERNATIONAL ORGANIZATION OF STANDARDIZATION. International vocabulary of basic and general terms in metrology. $2^{\text {nd }} \mathrm{ed}$. Geneva, Switzerland, 1993

4. De Bièvre; CITAC, Orlando, Florida, 1999.

5. INTERNATIONAL ORGANIZATION OF STANDARDIZATION. Statistics-Vocabulary and symbols - Part 1: Probability and general statistical terms. Geneva, Switzerland, 1993.

6. Ellison, S. L. R.; Williams, A.; Accred. Qual. Assur. 1998, 3, 6.

7. INTERNATIONAL ORGANIZATION OF STANDARDIZATION. Guide to the Expression of Uncertainty in Measurement. $1^{\text {st }}$ ed. Geneva, Switzerland, 1993.

8. EURACHEM. Quantifying uncertainty in analytical measurement. $2^{\text {nd }}$ ed., Helsinki, 2000.

9. da Silva, R. J. N. B.; Camões, M. F. G. F. C.; Barros, J. S.; Anal. Chim. Acta 1999, 393, 167.

10. Ríos, A.; Varcácel, M.; Accred. Qual. Assur. 1998, 3, 14

11. ANALYTICAL METHODS COMMITTEE; Analyst 1995, 120, 2303.

12. Horwitz, W.; Albert, R.; Analyst 1997, 122, 615.

13. Barwick, V. J.; Ellison, L. R.; Fairman, B.; Anal. Chim. Acta 1999, 394, 281.

14. Buchmann, J. H.; Tese de Doutorado; IPEN, São Paulo, SP, 2000.

15. Giaccomo, P.; Metrologia 1981, 17, 73.

16. Scarmínio, J.; Bruns, R.; Planejamento e otimização estatística nas medições; UNICAMP, Campinas, SP, 1997. 